\title{
El enseñar/ aprender de la Enfermería Comunitaria en la licenciatura
}

The teaching / learning of community nursing in undergraduates

Ensino / aprendizagem de grau Enfermagem Comunitária

DOI: http://dx.doi.org/10.23913/ride.v7i14.283

María Araceli Félix Amézquita

Escuela Superior de Enfermería Culiacán, Universidad Autónoma de Sinaloa, México aracelifelix56@hotmail.com

Jesús Roberto Garay Núñez

Escuela Superior de Enfermería Culiacán, Universidad Autónoma de Sinaloa, México

jrgarayn@hotmail.com

María Isabel Santos Quintero

Escuela Superior de Enfermería Culiacán, Universidad Autónoma de Sinaloa, México

misq@hotmail.com

\section{Resumen}

La presente investigación es un estudio cualitativo descriptivo. Objetivos: 1. Describir y analizar el enseñar/aprender de la Enfermería Comunitaria, en estudiantes de Licenciatura en Enfermería de la Escuela Superior de Enfermería en Culiacán, Sinaloa, México. 2. Analizar el enseñar/aprender en el proceso pedagógico. El abordaje teórico se basó en Pedro Demo, Díaz Barriga, Vera Waldow y Vilma de Carvalho. Se aplicó entrevista semi-estructurada a docentes y estudiantes. Se respetaron los principios éticos y criterios de rigor científico. Se utilizó el análisis de contenido temático, surgiendo tres categorías Temáticas: 1. El enseñar/aprender individual de la Enfermería Comunitaria. 2. El enseñar/aprender colectivo/grupal de la Enfermería Comunitaria. 3. Complementado en la práctica el enseñar/aprender individual con el colectivo/grupal, de la Enfermería Comunitaria. 
Conclusiones: Dadas las condiciones actuales caracterizadas por los cambios científicos y tecnológicos, el enseñar/aprender individual es importante más no suficiente, principalmente en el ámbito comunitario que es un espacio de socialización y de trabajo interdisciplinario. Es necesaria la interacción con los "otros", el profesor y los estudiantes entre sí, que facilita el aprendizaje tanto en lo individual como en lo colectivo; además de tomar la práctica en escenarios reales como espacios para la reflexión- acción, para el desarrollo de procesos de cuidar.

Palabras clave: enseñanza, aprendizaje y praxis de Enfermería.

\section{Abstract}

The present investigation is a qualitative descriptive study, objectives: 1. Describe and analyze the teaching / learning of community nursing in nursing undergraduate students of the School of Nursing in Culiacan, Sinaloa, Mexico. 2. Analyze the teaching/learning in the educational process. The theoretical approach was based on Pedro Demo, Diaz Barriga, Vera Waldow y Vilma de Carvalho. Teacher and students responded a semi-structured interview. The Ethics principles and scientific criteria were respected. The thematic content analysis was used, emerging three thematic categories: 1. The individual's teaching / learning of community nursing. 2. The collective / group teaching / learning of community nursing. 3. Supplemented in practice the individual teaching / learning with the collective/group of community nursing.

Conclusions: In the current conditions characterized by technological and scientific changes, the individual teaching / learning is important but not sufficient mainly at the community level since it is a space for socialization and interdisciplinary work. Interaction with the "others", teacher and students together, which facilitates learning both individually and collectively, in addition to taking, practice in real scenarios as spaces for reflection-action, for the development of caring processes.

Key words: teaching, learning and nursing praxis. 


\section{Resumo}

Esta investigação é um estudo descritivo qualitativo. Objetivos: 1. Descrever e analisar o ensino / aprendizagem de Enfermagem Comunitária em estudantes de Enfermagem da Escola de Enfermagem em Culiacan, Sinaloa, México. 2. Analisar o ensino / aprendizagem no processo educacional. A abordagem teórica foi baseada em Pedro Demo, Diaz Barriga, Waldow Vera e Vilma de Carvalho. professores entrevista semi-estruturada e estudantes aplicado. princípios e critérios de rigor científico éticos é respeitada. a análise de conteúdo temática foi utilizada, surgindo três categorias temáticas: 1. O ensino / aprendizagem de Enfermagem Comunitária individualmente. 2. O ensino / aprendizagem coletiva / grupo de enfermagem comunitária. 3. Complementado na prática, o ensino / aprendizagem individualmente com o / grupo coletivo de enfermagem comunitária.

Conclusões: dadas as atuais condições caracterizadas pela mudança científica e tecnológica, ensino / aprendizagem individualmente importante, mas insuficiente, especialmente ao nível da comunidade é um espaço de socialização e trabalho interdisciplinar. interação com "outros", o professor e os alunos juntos, o que facilita a aprendizagem tanto individual como coletivamente é necessário; Além de tomar prática em cenários reais como espaços de processo de desenvolvimento de reflexão-ação de cuidados.

Palavras-chave: ensino, aprendizagem e prática de enfermagem.

Fecha Recepción: Mayo $2016 \quad$ Fecha Aceptación: Octubre 2016

\section{Introducción}

Las Instituciones de Educación Superior y de Enfermería en particular, en la última década se han preocupado por la calidad y la permanente actualización de los programas de estudios, para que la formación de los profesionales sea pertinente a las exigencias de un mundo en constante cambio. 
En este sentido, la universidad autónoma de Sinaloa en correspondencia la escuela de Enfermería han adoptado el modelo curricular por Competencias Profesionales Integradas, que privilegia el aprendizaje y el papel activo del alumno. En el centro del proceso educativo está el estudiante, que es quien debe desarrollar habilidades que le faciliten su aprendizaje. El profesor deja de ser el centro, para convertirse en mediador entre el alumno y el conocimiento.

De acuerdo a lo anterior, la enseñanza se concibe como un proceso dinámico, participativo e interactivo entre el educador y educando, en las que las tareas del docente se enfocan a dirigir el proceso autónomo y consciente del estudiante en la construcción de sus conocimientos, habilidades y valores; el enseñar no solo implica proporcionar información, sino ayudar a aprender y a desarrollarse como personas. La meta de la actividad del docente es incrementar la competencia, la comprensión y la actuación autónoma de sus estudiantes (Díaz Barriga, 2010: 5).

Es importante dejar los modelos de enseñanza con énfasis en los contenidos y en el desarrollo de determinadas habilidades y comportamientos (los cuales puedan ser medidos) que consecuentemente inhiben la creatividad y el pensamiento independiente y crítico del estudiante (Waldow, 2008: 178-179).

En relación a la formación en Enfermería, Carvalho (2009) menciona que el modelo de enseñanza deberá formar una conciencia crítica, una nueva mentalidad profesional en consonancia con los significados del arte de la Enfermería y el cuidado de la salud, en el contexto de la nueva visión sobre ver las personas enfermas o sanas con necesidades de atención, medidas de apoyo y las instrucciones para las enfermeras especialmente capacitadas, para ayudar a las personas, familias y comunidades en la recuperación de la salud y el mantenimiento de la vida (p: 6).

Es así que la Enfermería Comunitaria es un elemento fundamental de los programas universitarios de la profesión. Las y los enfermeros egresados deben estar preparados para proporcionar el cuidado de Enfermería, a las personas sanas o enfermas en los diferentes escenarios, y estar preparados para actuar en escenarios políticos y económico-sociales, rápidamente cambiantes que influyen en la prestación de los servicios y cuidados de salud. 
Congruente con lo anterior en plan de estudios de licenciatura en Enfermería de esta Unidad Académica, se incluyen materias teóricas y prácticas enfocadas al primero, segundo y tercer nivel de atención, contemplando unidades de aprendizaje como: Enfermería Comunitaria, Epidemiología, Salud Pública, Medicina Preventiva, así como la Práctica Comunitaria a partir del segundo semestre de la carrera. Sin embargo se observa con frecuencia que los estudiantes no muestran gran interés por la Atención Comunitaria, se enfocan principalmente a los aspectos curativos ocupándose poco de la promoción de la salud y la prevención de enfermedades.

Con base en esta problemática se plantea esta investigación, que implica reflexionar sobre los componentes que se involucran en el proceso de enseñanza y aprendizaje, que incluye al sujeto cognoscente, al profesor y al contenido programático. El objetivo de este estudio es: describir y analizar el enseñar/aprender de la Enfermería Comunitaria, en estudiantes de licenciatura de la Escuela Superior de Enfermería Culiacán, Sinaloa, México.

\section{Material y método}

El presente trabajo de investigación es un estudio cualitativo descriptivo, cuyo objetivo fue describir y analizar el enseñar/aprender de la Enfermería Comunitaria, en estudiantes de licenciatura de la Escuela Superior de Enfermería Culiacán, Sinaloa, México. La investigación cualitativa responde a cuestiones muy particulares, se preocupa al igual que las Ciencias Sociales, por los fenómenos educativos, como una realidad que no puede ser cuantificada. Busca describir, comprender e interpretar los fenómenos, a través de las percepciones y significados producidos por las experiencias de los participantes (Hernández, 2014:11).

Los sujetos de investigación fueron 11 docentes y 11 estudiantes de la Escuela Superior de Enfermería Culiacán. Se seleccionaron los docentes que imparten la materia de Enfermería Comunitaria, del turno matutino y vespertino, e instructoras de Clínicas Comunitarias de la Escuela Superior de Enfermería Culiacán. Se seleccionaron estudiantes del $3^{\circ}$ al $8^{\circ}$ semestre de la carrera, en ambos turnos (matutino y vespertino). Esto debido a que son estudiantes que ya han cursado las materias de Enfermería Comunitaria, Epidemiologia, Salud Pública, y han tenido la experiencia de prácticas hospitalarias y comunitarias en estas áreas. 
Para recabar la información, se aplicó una entrevista semi-estructurada a los docentes seleccionados y otra a los estudiantes participantes. Utilizando el criterio de la saturación de datos, esto es, hasta que ya no se obtiene nueva información y ésta comienza a ser redundante.

En la entrevista al profesor se incluyeron los datos en primer lugar que permiten su caracterización como: edad, sexo, nivel de instrucción, experiencia en el área asistencial y en el área docente, y en la segunda parte, los datos específicos que corresponde a la metodología de enseñanza y aprendizaje. Para proteger la confidencialidad de los informantes, se utilizaron seudónimos, acompañados de algunas siglas asignadas junto a los seudónimos; la letra E= (Estudiante), EP= (Estudiante de Práctica), D (Docente), DP (Docente de Práctica).

Posterior a la recolección de información, se realizaron los análisis de contenido y temático de los datos obtenidos en las entrevistas. El análisis de contenido consistió en un conjunto de procedimientos interpretativos de productos comunicativos (mensajes. textos o discursos) que proceden de procesos singulares de comunicación previamente registrados. Para demostrar la rigurosidad de la investigación se recurrió a la auditabilidad, confirmabilidad y a la triangulación de los datos.

\section{Resultados y discusión}

A partir de los discursos de docentes y estudiantes entrevistados, surgieron tres Categorías Temáticas: 1. El enseñar/aprender individual de la Enfermería Comunitaria. 2. El enseñar/aprender, colectivo/grupal de la Enfermería Comunitaria. 3. Complementado en la práctica el enseñar aprender individual con el colectivo/grupal de la Enfermería Comunitaria.

\section{El enseñar/aprender individual de la Enfermería Comunitaria.}

Existen diversas estrategias de enseñanza, que se pueden utilizar de manera reflexiva para facilitar el aprendizaje significativo de los estudiantes en los diferentes momentos del proceso de enseñanza y aprendizaje; las metodologías individuales de trabajo ofrecen al estudiante la oportunidad de desenvolverse y desarrollar sus potencialidades personales. En 
las tareas y trabajos individuales el estudiante hace uso de sus habilidades de pensamiento como: la observación, comparación, análisis, síntesis y el razonamiento.

En Enfermería Comunitaria se utilizan metodologías individuales para el aprendizaje. Así se evidencia en los discursos de los estudiantes entrevistados:

[...] "Para mi aprendizaje en la materia de Enfermería Comunitaria, me baso más en la lectura... hago lectura previa del tema al realizar la lectura, hago reflexión, siempre me ha funcionado”. [...] (E1-Jom)

[...] "Las estrategias que utiliza la maestra para nuestro aprendizaje son...resúmenes, lecturas y debates”. [...] " (E2-Juan)

[...] "Para el aprendizaje en la materia de Enfermería Comunitaria realizamos trabajos individuales como lecturas, resúmenes del tema, mapas conceptuales, lluvia de idea” [...] (E3-Gemela)

En este sentido, Waldow señala que el proceso de aprender, es una actividad individual que se desarrolla en un contexto social y cultural. Es el resultado de procesos cognitivos individuales (hechos, conceptos, procedimientos, valores) con los que se construyen nuevas representaciones mentales (conocimientos), que posteriormente pueden aplicarse en situaciones diferentes a los contextos en los que aprendieron (Waldow, 2008: 184).

Los docentes estudiados en el aula de clases, así como en la práctica de Enfermería Comunitaria, también manifiestan que utilizan metodologías de enseñanza individuales, que privilegian la participación del estudiante en su proceso de aprender. Así se muestra en los siguientes discursos de los profesores.

[...] "En la materia de Enfermería Comunitaria los alumnos realizan lecturas previas de su antología, resúmenes, investigación del tema y participación individual” [...] (D1-Elena)

[...] "Los métodos y estrategias didácticas que utilizo para la enseñanza de la materia de Enfermería Comunitaria son: lluvia de ideas, participaciones y resúmenes." [...] (D2-Lucy) 
[...] "En la sesión de clase utilizamos lluvia de ideas, lectura comentada, y pido resúmenes, para que el estudiante retroalimente lo que se vio en la clase”. [...] (D5- José)

[...] "La guía de valoración la aplican individualmente...toman signos vitales...realizan una narrativa sobre su experiencia". [...] (DP5-María)

El empleo de una metodología de trabajo individual, es importante en su proceso de aprender, ya que el enfrentarse a una situación que le exija la resolución de una determinada tarea, puede hacer que el estudiante se motive y utilice todas sus capacidades para la resolución de dicha tarea, y por ende, contribuir a su aprendizaje. Sin embargo, en muchas ocasiones para lograr el aprendizaje, se requiere el acompañamiento o ayuda de otra persona, como puede ser, algún compañero más competente.

\section{El enseñar/aprender, colectivo/grupal de la Enfermería Comunitaria.}

Las estrategias grupales o en equipo son fundamentales, principalmente cuando el alumno no logra realizar una tarea por sí mismo. El trabajo colectivo-grupal promueve no solo la construcción de conocimientos, sino el desarrollo de valores como la cooperación, la comunicación y la responsabilidad del equipo de trabajo.

La cooperación en la actividad grupal consiste en trabajar juntos para alcanzar objetivos comunes. En una situación cooperativa, los individuos procuran obtener resultados que son beneficiosos para ellos mismos y para todos los miembros restantes del grupo. El aprendizaje cooperativo es el empleo didáctico de grupos reducidos en los que los alumnos trabajan juntos para maximizar su propio aprendizaje y el de los demás (Johnson, en Díaz Barriga y Hernández, 2010:90).

Los docentes entrevistados utilizan metodologías grupales en el proceso de enseñanzaaprendizaje, tanto en el aula de clases como en la práctica. Así se expone en los siguientes discursos.

[...] "En la materia de Enfermería Comunitaria los alumnos, trabajan en equipos se hacen comentarios de manera grupal y exposiciones utilizando Power Point." [...] (D1-Elena) 
[...] "Los métodos y estrategias didácticas que utilizo para la enseñanza de la materia de Enfermería Comunitaria son: trabajo en equipo, exposiciones grupales utilizando Power Point y carteles. "[...] (D2-Lucy)

[...] "En la materia de Enfermería Comunitaria trabajo en equipo, con la participación activa de cada uno de los alumnos, los motivo mucho a expresarse verbalmente, que aprendan a comunicarse con los demás, además manejo mucho la lectura y el análisis. "[...] (D3- Lupita)

Los docentes de prácticas comunitarias en sus discursos manifiestan también utilizar metodologías grupales para el enseñar/aprender.

[...] "En la práctica el alumno se distribuye por equipos de cinco integrantes...hacen la visita domiciliaria, se levanta el censo poblacional del grupo etáreo con el que se va a estudiar.” [...] (DP1-Anel)

[...] "Los muchachos trabajan en equipo... en equipo, aplican la guía pediátrica y la guía familiar, con base a esas guías vamos programando las actividades". [...] (DP2-Ana)

Los estudiantes convergen con lo señalado por los docentes, así lo manifiestan:

"Los métodos y estrategias que utiliza el docente para la enseñanza son: la lectura, la reflexión y las exposiciones, docente y el estudiante utilizando proyector". [...] (E1-Jom)

[...] "Las estrategias que utiliza la maestra para nuestro aprendizaje son: exposiciones tanto de la maestra como por equipos, se fomenta la participación de todo el grupo. "[...] (E2-Juan)

En este sentido Demo (2009) señala que es esencial aprender a trabajar juntos, lo que implica que cada miembro del grupo debe presentar una contribución individual elaborada. En el trabajo colectivo cada miembro del grupo debe funcionar correctamente para combinar la originalidad individual, con la exigencia de la cooperación colectiva. El proyecto 
colectivo debe ser la combinación de las contribuciones individuales, no de los vacíos individuales (p. 4).

El trabajo en equipo es fundamental en el proceso de aprender, En el trabajo grupal se da una interacción entre los miembros en donde se comparte la responsabilidad de la tarea, y con el aporte de todos se soluciona y se construye el conocimiento. Esto permite desarrollar habilidades personales y sociales en el estudiante.

Para el trabajo grupal son determinantes los procesos psicológicos individuales de cada uno de los integrantes del grupo, como la atención, memoria percepción y el razonamiento, los cuales son indispensables para el desarrollo de tareas complejas.

\section{Complementado en la práctica el enseñar/aprender individual con el colectivo/grupal de la Enfermería Comunitaria.}

El trabajo individual y colectivo puede ser complementado en la práctica, que se constituye en un espacio para el aprendizaje contextual, basado en la realidad; el docente pone al estudiante en contacto con situaciones de la realidad profesional, que deberá resolver integrando sus conocimientos, al brindar cuidado al individuo, familia y comunidad en los diferentes contextos.

En la enseñanza situada, se pone énfasis en el aprendizaje basado en la experiencia en contextos reales, donde los estudiantes realizan actividades auténticas, significativas, en las que se busca desarrollar conocimientos y habilidades propios de la profesión, así como la participación en la solución de problemas (Díaz Barriga 2010: 38 y 39).

Los docentes entrevistados, manifiestan utilizar metodologías de enseñanza encaminadas a complementar en la práctica el trabajo individual y colectivo, sin embargo otros mencionan que no les alcanza el tiempo para hacerlo. Esto se evidencia en los siguientes discursos:

[...] "Las estrategias didácticas que utilizo son: enriquecer el conocimiento con la práctica, llevarlos al laboratorio, a un escenario real, hacer visitas a la comunidad, hacer un recorrido, realizar matrices y encuestas". [...] (D4Rosy) 
[...] "Otra estrategia que utilizo es asistir al laboratorio donde los muchachos integran lo teórico a la práctica, llevando a cabo el programa de inmunizaciones, en forma personalizada los estudiantes explican para qué sirve la vacuna, dosis, indicaciones, contraindicaciones”. [...] (D1-Elena)

[...] "La unidad de aprendizaje de Enfermería Comunitaria contempla horas teóricas y horas prácticas en estas últimas se realizan simulacros, socio dramas y vamos a la comunidad a elaborar el croquis. Realizo talleres sobre vacunas, programas prioritarios y diagnóstico de salud...en los que participan en forma individual y en equipo". [...] (D3-Lupita)

[...] "Algunos temas son muy extensos, como el tema de vacunas, los lleve solo un día al laboratorio a que se enseñen a aplicar las vacunas, las técnicas de aplicación intradérmica y subcutánea ya lo vieron en el primer semestre... En el aula nada más se da la teoría no hacemos la práctica... en la comunidad se le va enseñar...No nos alcanza el tiempo, vemos los temas muy de prisa”. [...] (D2-Lucy)

[...] "La enseñanza de la unidad de aprendizaje de Enfermería Comunitaria solo se realiza en el espacio del aula de clases... Lo ideal sería que como docentes lleváramos a los alumnos a una comunidad antes de que se vallan a la práctica, para que vean realmente sus características”. [...] (D5-José)

Carvalho (2009) menciona que en el área de la Enfermería, los estudiantes necesitan aprender a través de sus propias experiencias. La búsqueda de la respuesta coherente a la realidad, requiere el compromiso personal y el compromiso de conocimiento inherente de la materia. Que puedan asimilar el por qué y el cómo hacer frente a su objeto de estudio o trabajo, que son los cuidados de Enfermería. Sólo en las dimensiones de una práctica viva los estudiantes de Enfermería aprenderán estrategias de intervención y relevancia de la decisión para la Enfermería, para ello, tienen que encontrarse cara a cara con las situaciones problema (p. 6). 
La toma de decisiones implica elegir una opción entre las disponibles, para resolver un problema real o potencial, esto requiere que la persona haga uso de su razonamiento y de un pensamiento crítico reflexivo para elegir la mejor solución a los problemas que se le presenten en el ámbito profesional.

La práctica ofrece al estudiante esa posibilidad de encontrarse y actuar en la realidad, tomando la teoría y práctica como dos momentos consecutivos, complementarios, que no pueden ser separados en la acción didáctica, la teoría se puede fortalecer a partir de la práctica y la práctica se sustenta en teoría, y viceversa, no como un proceso lineal, sino circular; como un ir y venir entre la teoría y la práctica. Se trata que el alumno encuentre significados que fortalezcan su proceso de aprendizaje. En el aprendizaje de Enfermería es importante el desarrollo de la teoría y la práctica de una forma simultánea y sistematizada, como un eje fundamental para el desarrollo de habilidades y destrezas profesionales.

Los estudiantes coinciden en lo mencionado por los docentes: las metodologías de enseñanza se orientan a complementar en la práctica el trabajo individual y colectivo; así lo manifiestan:

[...] "En la materia de Enfermería Comunitaria también asistimos al laboratorio de la escuela, vamos a la comunidad a practicar según lo que hayamos visto en el aula... hacemos la elección de un tema y exponemos en un rotafolio, en tríptico y se lo damos a la comunidad sobre el tema que nos tocó”. [...] (E2- Juan)

[...] "Salimos a la colonia a aplicar lo visto en la teoría... lo que tenemos que hacer en la comunidad...en equipos o en parejas realizamos el croquis, la guía de observación, entrevista... aplicamos los instrumentos que se tienen que aplicar en la práctica comunitaria." [...] (E3-Gemela)

La práctica es un espacio formativo, de reflexión, durante su proceso de aprendizaje, los valores y las actitudes representan los fundamentos básicos para su formación. El profesor no solo enseña, se convierte en modelo para el estudiante, que aprende de él las actitudes y valores que manifiesta en su actuar. Especialmente cuando se forman profesionales que van 
a desarrollar sus actividades directamente relacionadas con las necesidades de las personas en su contexto. Es así que el saber cuidar se relaciona con las formas de aprender.

En los discursos de los docentes comunitarios también se evidencia que el enseñar/aprender se dirige a complementar el aprendizaje individual-colectivo en la práctica.

[...] "En la práctica, hacemos juntos actividades...Utilizo la enseñanza vivencial personalizada...enseñarle en la vivencia en la casa o en la comunidad o en la manzana, estar con ellos". [...] (DP1- Anel)

[...] "Para en la práctica comunitaria, los muchachos aplican la guías... para enseñarles a aplicarlas, nos reunimos en grupo, vamos leyendo y se va explicando donde tengan dudas y sobre los términos que desconocen... cuando aplican la guía estoy con ellos y si surgen dudas se aclaran inmediatamente... aunque no podemos estar con ellos siempre...ellos tienen que hacer las cosas por sí mismos para que puedan ir aprendiendo...en el punto de reunión se revisan y se aclaran dudas". [...] (DP2- Ana)

[...] "En las prácticas, se dan pláticas y se hace una feria de la salud... Les enseño a los muchachos a aplicar las guías como nos enseñaron en la escuela a aplicarlas sistemáticamente... primero vemos las guias las leemos y explicamos... yo hago la primera entrevista para que se den cuenta los muchachos como se hace”. [...] (DP3- Esaú)

La formación en la práctica reflexiva, postula la experiencia de aprender haciendo mediante una buena acción tutorial; los estudiantes aprenden las formas de arte profesional mediante la práctica de hacer o ejecutar reflexivamente aquello en lo que buscan convertirse en expertos, que resultan esenciales a su competencia académica, personal o profesional. Se les ayuda a llevarlos a cabo gracias a la mediación que ejercen sobre ellos "otros" más experimentados que usualmente son los profesores o formadores (Díaz Barriga 2010: 12).

La enseñanza a través de la reflexión en la acción, se constituye como un método eficaz particularmente en Enfermería. Una condición básica en el aprendizaje practico, es el dialogo entre docente y estudiante, ya que el maestro trasmite mensaje a sus aprendices tanto 
en forma verbal como en la forma de ejecución; el alumno reflexiona sobre lo que escucha o ve hacer al docente, este ejecuta las prescripciones del docente a través de la imitación reflexiva, derivada del modelado del maestro. La calidad del aprendizaje depende en gran medida de la habilidad del docente para adaptar su demostración y su descripción a las necesidades cambiantes del alumno (Schón en Díaz Barriga, 2010: 13).

En una enseñanza centrada en el cuidado; tanto la clase, el laboratorio, como el campo clínico se tornan en lugares de encuentros. Es necesario sensibilizar y concientizar a los alumnos de que el aprendizaje es un proceso sin fin; deben ser capacitados para buscar medios a fin de mantenerse actualizados, perfeccionando su conocimiento. Esto equivale a empoderar al alumno para aprender. Además el pensamiento crítico es un instrumento valioso, que los profesores deben desarrollar en sus alumnos (Waldow, 2009:9).

Los estudiantes en sus discursos divergen en algunos puntos, con lo que manifiestan los docentes comunitarios:

[...] "En la práctica aplicamos las guías, listados de viviendas, hacemos diagnósticos, para aprender leemos el programa, la docente nos explica las actividades a efectuar y después de la explicación vamos a las viviendas a realizarlas... la maestra se queda en el punto de reunión... no nos acompaña”. [...] (EP1- María)

[...]En la práctica aplicamos la guía de observación, listado de viviendas, guía de valoración, tomamos signos vitales...nos piden planes de cuidados, planes de charla y trípticos y rotafolios...En el punto de reunión la docente explica cómo llenarlos, nos revisa, en ocasiones va a revisar que apliquemos las guías". [...] (EP3- Sofía)

[...] "En la práctica...en la escuela la docente de aula nos enseña las guías y la profesora de práctica antes de ir a aplicarlas a las familias, en el punto de reunión, nos explican y nos aclaran dudas... vamos, las aplicamos... se quedan esperándonos en el punto de reunión y en ocasiones si tenemos dudas, les decimos la ubicación de donde estamos y van y nos ayudan." [...] (EP5-Brianda) 
En una enseñanza centrada en el cuidado, la orientación y el apoyo del profesor son transcendentales. El docente permite que los alumnos encuentren sus propios medios para perseguir sus proyectos, pero ayudando siempre que sea necesario. Esa ayuda se practica en el sentido de orientar, iluminar, motivar, sensibilizar, e incentivar la búsqueda, la imaginación, la creatividad. También ayuda a los alumnos proveyendo materiales y recursos de forma que sean expuestos a experiencias estimuladoras y significativas (Waldow, 2009:6).

El aprendizaje de contenidos prácticos ocurre en etapas, primero: la apropiación de datos relevantes respecto a la tarea. Esta etapa se centra en proporcionar al aprendiz la información o conocimiento factual relacionado con el procedimiento y las tareas a desarrollar. Y posteriormente la actuación o ejecución del procedimiento, donde al inicio el aprendiz procede por ensayo y error, mientras el docente lo va corrigiendo mediante episodios de práctica con retroalimentación. En esta fase, se llega a manejar un doble código declarativo y procedimental que debe culminar con la fijación del procedimiento. Una creencia errónea muy arraigada es que es posible ejecutar un procedimiento a partir de dar al alumno la información teórica o las reglas de cómo hacerlo, sin ofrecer una retroalimentación (Díaz Barriga, 2010: 44).

Por lo anterior, el acompañamiento del docente en el proceso de construcción de conocimiento, principalmente hablando en la práctica de Enfermería es fundamental; las situaciones de cuidado, las experiencias y las actividades que se seleccionen deben contribuir a provocar en los estudiantes la reflexión, y la transformación en sus capacidades, conocimientos y competencias. Es importante que el docente deje de considerarse a sí mismo solo como un supervisor que inspecciona el trabajo del estudiante, para que pase a concebirse como un facilitador que implica hacer fácil y posible la ejecución del cuidado, a través de guiar, compartir experiencias, proporcionar su apoyo, colaboración y retroalimentación. 


\section{Conclusiones}

A partir de los discursos de estudiantes y docentes, se puede afirmar que los docentes de Enfermería Comunitaria han comenzado a dar al estudiante un rol participativo, activo en su proceso de aprender, sin embargo, dadas las condiciones actuales caracterizadas por los cambios científicos y tecnológicos, se requiere desarrollar formas de enseñar y de aprender diversas, acordes a la realidad actual. El enseñar/aprender individual es importante más no suficiente, principalmente en el ámbito comunitario, que es un espacio de socialización y de trabajo interdisciplinario. Es necesaria la interacción con los "otros", el profesor y los estudiantes entre sí, que facilita el aprendizaje tanto en lo individual como en lo colectivo, además de tomar la práctica en escenarios reales como espacios para la reflexión- acción. Se requiere admitir que el principal objetivo del docente debe ser el enseñar a aprender.

El aprendizaje en las prácticas, especialmente en la práctica comunitaria se realiza en un contexto diferente al académico y en condiciones muy particulares; el estudiante se enfrenta a una realidad donde el conocimiento teórico adquirido en el aula es el marco de referencia que fundamenta sus acciones. El reto es que ese cierto "rechazo" que los estudiantes muestran por lo teórico, por considerarlo innecesario en la práctica o alejado de la realidad, cobre verdadero sentido y significado, además, que se sensibilice al estudiante para el desarrollo de procesos de cuidar como una actividad reflexiva, tomando en cuenta que el proceso salud-enfermedad no corresponde únicamente al ámbito biológico, sino que es influenciado por factores sociales, económicos y políticos, por tanto, asumir que la responsabilidad profesional no sólo está en el ámbito clínico, sino también en la comunidad. Ir más allá de concebir el cuidado de la persona en fase de enfermedad, también para el fomento de la salud, prevención de enfermedades y el mantenimiento de la vida. 


\section{Bibliografía}

Carvalho, V. (2009) Por uma epistemologia do cuidado de enfermagem e a formação dos sujeitos do conhecimento na área da enfermagem - do ângulo de uma visão filosófica. Esc Anna Nery Rev Enferm abr-jun; 13 (2): 406-14. Recuperado de [www.redalyc.org/articulo.oa?id=127715322024]

Consejo Internacional de Enfermería - CIE (2013) Recuperado de [www.cge.enfermundi.com/servlet/Satellite?cid=1102684712930...]

Corrales, V. (2009) Plan Institucional de Desarrollo Visión 2013. Universidad Autónoma de Sinaloa.

Demo, P. (2007) Educar pela pesquisa; Horizontes reconstructivos, Brasil: $\quad$ Editores asociados.

Demo, P. (2008) Habilidades do Século XXI. [www.senac.br/bts/342/artigo-1.pdf...] 5 Febrero 2016

Hernández S. R. (2010) Metodología de la investigación. México, ed. Mc Graw Hill.

Díaz Barriga, F., y Hernández, G. (2010) Estrategias docentes para el aprendizaje significativo. Una interpretación constructivista. México: Mc Graw Hill.

DEMO, P. (2009) Ser professor é cuidar que o aluno aprenda. Porto Alegre: Mediação. Recuperado de [https://www.estantevirtual.com.br/.../pedro-demo/ser-professor...c.]

Martínez, M. (2014) Ciencia y arte en la metodología cualitativa. México: Trillas.

Waldow, V. (2008) Cuidar: expresión humanizadora de la Enfermería. México: palabra.

Waldow, V. (2009) Enseñanza de Enfermería centrada en el cuidado. Vol. 9, Núm. 3, diciembre-Universidad de La Sabana Colombia. Recuperado de [www.redalyc.org/articulo.oa?id=74112147005] 